\title{
Status of Tuberculosis in South Korea and the Need for a New Method to Diagnose Active Tuberculosis
}

\author{
So Hee Dho' ${ }^{1}$, Kyungjong Kim² ${ }^{2}$, Sung Hee Jung ${ }^{1}$ and Jae Cheong Lim*1 \\ ${ }^{1}$ Radioisotope Research Division, Department of Research Reactor Utilization, Korea Atomic Energy Research Institute, Daejeon 34057 , Republic of Korea
}

${ }^{2}$ Department of Research and Development, Korean Institute of Tuberculosis, Cheongju-si, Chungcheongbuk-do, Korea

Received: May 30, 2018; Published: June 05, 2018

*Corresponding author: Jae Cheong Lim, Radioisotope Research Division, Department of Research Reactor Utilization, Korea Atomic Energy Research Institute, Daejeon 34057, Republic of Korea

\begin{abstract}
Tuberculosis (TB) is a global health concern even in South Korea. TB incidence in South Korea is the highest among Organisation for Eoconomic Co-operation and Development (OECD) countries, and is supposed to be due to the Korean War in 1950. TB has not yet been eradicated, and is the most common infection and death among infectious diseases in South Korea. Therefore, there is a need for a method to diagnose active tuberculosis quickly and inexpensively on site, and the detection of mycobacterial proteins in sputum using a radioimmunoassay or membrane filtration method can be an alternative. In addition, the newly developed diagnostic method can contribute greatly to Africa, Southeast Asia, and North Korea, which have become major problems with tuberculosis.
\end{abstract}

Abbreviations: OECD: Organisation for Eoconomic Co-operation and Development; KCDC: Korea Centers for Disease Control

\section{Mini Review}

\section{Tuberculosis (TB)}

TB caused by Mycobacterium tuberculosis is a global health concern for both developing and developed countries, which is approximately one-third of the world's population infected [1]. According to the World Health Organization Tuberculosis Report of 2016, the number of tuberculosis deaths was estimated to be 1.4 million out of the 2015 non-HIV infected. In addition, it was estimated that 390,000 of HIV-infected people died of tuberculosis. Tuberculosis is among the top 10 causes of death worldwide, and more deaths were made than HIV or AIDS among infectious disease. In particular, Africa, Southeast Asia, and North Korea accounted for more than $86 \%$ of all tuberculosis deaths [2].

\section{TB incidence in South Korea}

TB incidence in South Korea is the highest among Organisation for Eoconomic Co-operation and Development (OECD) countries. The TB incidencein south Korea was 7 times higher than the average for member countries of the OECD [3]. The major reason for the high incidence is the Korean War from 1950 to 1953. Because of malnutrition, overcrowding, and a lack of access to health care, the number of deaths from TB increaed drastically after the Korean War. The TB death rate was 5 -fold higher than that before the Korean War. In addition, the first effective antimicrobial drugs such as isoniazid had been developed by the 1950s. It was not until 1955 that the Korean National Center for Tuberculosis began the first official distribution of anti-TB drugs [4]. Despite the growing economy and continuous national efforts to control TB, South Korea has a disproportionately high burden of TB compared with most high-income countries [5]. The reasons for the high burden of TB in South Korea are a high prevalence of latent TB infection in the elderly population, an increasing population with diabetes, a high smoking rate, inadequate patient management, and immigrants from high-burden countries [6]. New tuberculosis patients, which have been on the rise since 2003, began to decline, peaking 2011. In 2017, the overall new tyberculosis notification rate was 55.0 per 100,000 people in South Korea. In particular, the incidence of tuberculosis in elderly people over 65 years old is $41.9 \%$. Because TB can be fatal to the elderly with weak immunity, effective measures for eradicating TB are needed [7]. As tuberculosis is the most common infection and death among infectious diseases in South Korea, national management is being carried out through the KCDC (Korea Centers for Disease Control).

\section{Need for New Methods to Diagnose Active TB in South Korea}

Early diagnosis of an active disease is essential for eradication of an epidemic as well as effective patient management. For erad- 
ication of TB, early diagnosis of active TB is the most important. Sputum is the most common specimen obtained for the diagnosis of active TB, and is directly tested through a microscopic examination or nucleic acid amplification tests. A direct microscopic examination is a fast and inexpensive method to identify acid-fast bacilli, but its sensitivity is poor [8]. For these reasons, WHO recommends a biomolecular test such as nucleic acid amplification. Xpert MTB/ Rif (Cepheid, Sunnyvale, CA, USA) is the most efficient and suitable tool for a nucleic acid amplification test of TB, and provides the results in $<2 \mathrm{~h}$ [9]. Nevertheless, a culture remains the gold standard test for the diagnosis of TB, but it requires a long waiting time (2-6 weeks) owing to the slow growth rate of mycobacteria [10]. The reason why tuberculosis has not been eradicated in South Korea is presumed to be the difficulty in diagnosing active TB of patients who are alienated, poor, or homeless. A nucleic acid amplification test is the most appropriate for an immediate inspection of active TB on site. However, expensive equipment and a skilled inspector for the test are not available in general hospitals, with the exception of university hospitals in South Korea. Therefore, new tools for the diagnosis of active TB that can be performed on site are needed.

Methods for the diagnosis of active TB are continually evolving in order to achieve more rapid, less expensive, and accurate results. Although a number of molecular diagnostic tools have been developed, the detection of mycobacterial proteins in sputum is most likely to confirm active tuberculosis. For protein detection, a radioimmunoassay method can be useful because a large number of samples can be evaluated concurrently, and it does not take much time. A membrane filtration can also be useful because the concentration of sputum can amplify the sensitivity.

\section{Conclusion}

In South Korea, tuberculosis is prevalent in the elderly, and is the most common deadly infectious disease. This is presumably due to the difficulty in diagnosing the major infected persons with active tuberculosis. Therefore, there is a need for a method to di- agnose active tuberculosis quickly and inexpensively on site, and the detection of mycobacterial proteins in sputum using a radioimmunoassay or membrane filtration method can be an alternative. In addition, the newly developed diagnostic method can contribute greatly to Africa, Southeast Asia, and North Korea, which have become major problems with tuberculosis.

\section{Acknowledgement}

This work was supported by a Korea Atomic Energy Research Institute major project: Development of Radioisotope Production and Application Technology (525330-18), and by the National Research Foundation of Korea (NRF), funded by the Ministry of Science and ICT (NRF-2017M2A2A6A01071321).

\section{References}

1. WHO (2015) Global tuberculosis report 2015. Geneva, Switzerland.

2. WHO (2016) Global tuberculosis report 2016. Geneva, Switzerland.

3. World Bank (2015) Incidence of tuberculosis (per 100,000 people).

4. Korean National Tuberculosis Association (1998) A history of tuberculosis in Korea. The Association, Seoul, South Korea.

5. Janssens JP, Rieder HL (2008) An ecological analysis of incidence of tuberculosis and per capita gross domestic product. Eur Respir J 32(5): 1415-1416.

6. Kim JH, Yim JJ (2015) Achievements in and Challenges of Tuberculosis Control in South Korea. Emerg Infect Dis 21(11): 1913-20.

7. Wonseo P (2018) Tuberculosis notification status in Korea in 2017. Weekly Health and Disease 11(13): 401-406.

8. Marais BJ (2008) Use of light-emitting diode fluorescence microscopy to detect acid-fast bacilli in sputum. Clin Infect Dis 47(2): 203-207.

9. Ardizzoni E (2015) Implementing the Xpert (R) MTB/RIF Diagnostic Test for Tuberculosis and Rifampicin Resistance: Outcomes and Lessons Learned in 18 Countries. PLoS One 10(12): e0144656.

10. Asmar S (2015) A Novel Solid Medium for Culturing Mycobacterium tuberculosis Isolates from Clinical Specimens. J Clin Microbiol 53(8): 2566-2569.

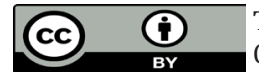

This work is licensed under Creative Commons Attribution 4.0 License

Submission Link: https://biomedres.us/submit-manuscript.php

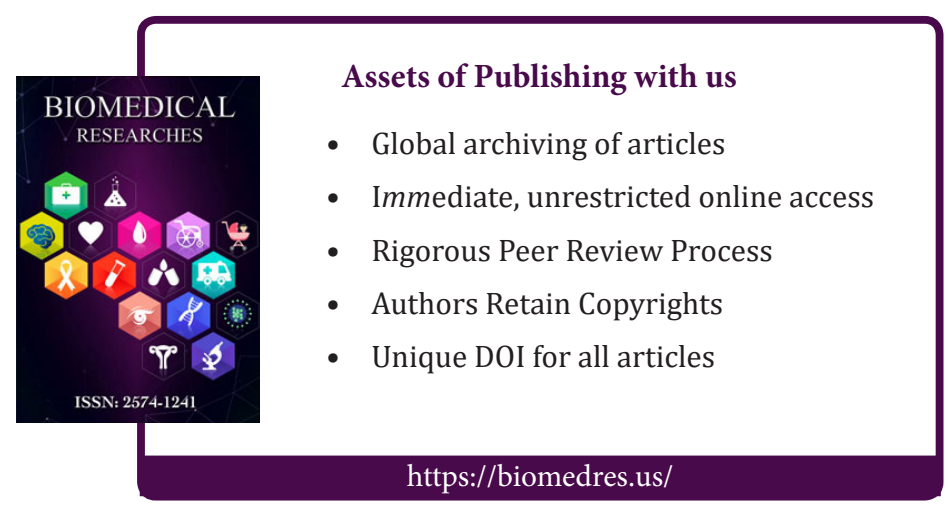

